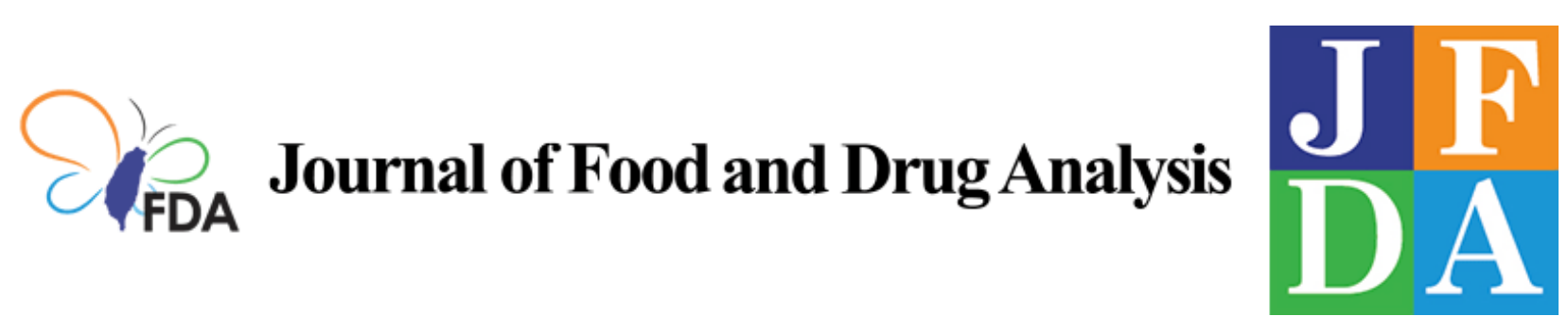

Volume 28 | Issue 4

Article 3

2020

\title{
Noble metal nanomaterial-based aptasensors for microbial toxin detection
}

Follow this and additional works at: https://www.jfda-online.com/journal

Part of the Food Science Commons, Medicinal Chemistry and Pharmaceutics Commons, Pharmacology Commons, and the Toxicology Commons

(c) (i) (8)

This work is licensed under a Creative Commons Attribution-Noncommercial-No Derivative Works 4.0 License.

\section{Recommended Citation}

He, Yue; Wen, Cong-Ying; Guo, Zhi-Jun; and Huang, Yu-Fen (2020) "Noble metal nanomaterial-based aptasensors for microbial toxin detection," Journal of Food and Drug Analysis: Vol. 28 : Iss. 4 , Article 3. Available at: https://doi.org/10.38212/2224-6614.1155

This Review Article is brought to you for free and open access by Journal of Food and Drug Analysis. It has been accepted for inclusion in Journal of Food and Drug Analysis by an authorized editor of Journal of Food and Drug Analysis. 


\title{
Noble metal nanomaterial-based aptasensors for microbial toxin detection
}

\author{
Yue He ${ }^{\mathrm{a}, *}$, Cong-Ying Wen ${ }^{\mathrm{b}}$, Zhi-Jun Guo ${ }^{\mathrm{c}}$, Yu-Fen Huang ${ }^{\mathrm{d}, \mathrm{e}, * *}$
}

\author{
${ }^{a}$ Laboratory of Quality \& Safety Risk Assessment for Citrus Products (Chongqing), Ministry of Agriculture, Citrus Research Institute, \\ Southwest University, Chongqing, 400712, PR China \\ ${ }^{\mathrm{b}}$ College of Science, China University of Petroleum (East China), Qingdao, 266580, PR China \\ ${ }^{\mathrm{c}}$ Department of Food Science and Engineering, Yanbian University, Yan Ji, 133002, PR China \\ ${ }^{\mathrm{d}}$ Institute of Analytical and Environmental Sciences, National Tsing Hua University, Hsinchu, 30013, Taiwan \\ e Department of Biomedical Engineering and Environmental Sciences, National Tsing Hua University, Hsinchu, 30013, Taiwan
}

\begin{abstract}
Microbial toxins generated by bacteria, fungi and algae cause serious food-safety problems due to the frequent contamination of foodstuffs and their poisonous nature. Becoming acquainted with the contamination condition of foodstuffs is highly dependent on developing sensitive, specific, and accurate methods for targeting microbial toxins. Aptamers, obtained from systematic evolution of ligands by exponential enrichment (SELEX), have significant advantages for microbial toxin analysis, such as small size, reproducible chemical synthesis, and modification, as well as high binding affinity, specificity, and stability. Besides, aptamers have a predictable structure and can be tailored using biomolecular tools (e.g., ligase, endonuclease, exonuclease, polymerase, and so on), which is conducive to the development of flexible and variable amplification methods. Recent studies revealed that the combination of aptamers and noble metal nanomaterials offers unprecedented opportunities for microbial toxin detection. Noble metal nanomaterials with outstanding physical and chemical properties facilitate the detection process and improve the sensitivity and specificity. In this review, we discuss current progress in the development of various noble metal nanomaterial-based aptasensors for microbial toxin detection. These noble metal nanomaterials include gold nanoparticles, gold nanorods, gold nanoclusters, silver nanoparticles, silver nanoclusters, and bimetallic nanomaterials. Aptasensors based on noble metal nanomaterials exhibiting high selectivity and sensitivity represent a promising tool for microbial toxin detection.
\end{abstract}

Keywords: Algal toxin, Aptamer, Bacterial toxin, Mycotoxin, Noble metal nanomaterial

\section{Introduction}

$\mathrm{F}$ ood contaminations caused by microbial toxins (such as bacterial toxin, mycotoxin, and algal toxin) are becoming serious problems to human health [1]. Among them, bacterial toxins have the highest significance with respect to public health. Bacterial toxins are generally classified into endotoxins and exotoxins. The most common bacterial toxins are Staphylococcal toxins, Clostridia botulinum and Clostridium difficile toxins,
Bacillus anthracis toxin, and Shiga toxins. Through the diet, bacterial toxins can interact with and damage host cells by disturbing the signaling pathways and structural integrity of the host cells, leading to various types of disease symptoms, such as nausea, vomiting, and diarrhea. Mycotoxins, being secondary toxic metabolites, are primarily derived from fungi, particularly Aspergillus, Penicillium, Fusarium, and Alternaria. They include hundreds of chemically toxic compounds, such as aflatoxins (AFs), ochratoxin A (OTA),

Received 31 May 2020; Received in revised form 4 July 2020; accepted 1 September 2020.

Available online 1 December 2020

* Corresponding author at. Laboratory of Quality \& Safety Risk Assessment for Citrus Products (Chongqing), Ministry of Agriculture, Citrus Research Institute, Southwest University, Chongqing, 400712, PR China. Fax: +86 2368349046.

** Corresponding author at. Institute of Analytical and Environmental Sciences, National Tsing Hua University, Hsinchu, 30013 , Taiwan. Fax: +88603 5752032 .

E-mail addresses: yuehe@cric.cn (Y. He),yufen@mx.nthu.edu.tw (Y.-F. Huang). 
trichothecene mycotoxin (T-2 toxin), zearalenone (ZEN), fumonisins (Fs), patulin (PAT), and so on. Toxicological research studies revealed that a long-lasting exposure to a low dose of mycotoxin is capable of causing strong carcinogenic, teratogenic, and mutagenic effects. Algal toxins are generated by algae and are found in both marine and freshwater environments. The consumption of seafood or water contaminated with algal toxins can cause serious disease symptoms, including tingling, nausea, vomiting, paralysis, and diarrhea. To anticipate potential risks and ensure food safety, it is highly demanded and crucial to develop selective, sensitive, rapid, low-cost and on-site detection techniques for microbial toxins.

To ensure the required detection performance, antibodies have long been the most popular recognition elements used for the binding of target analytes. Unfortunately, antibodies suffer from short shelf-life, high costs of manufacturing, relatively low stability and nonnegligible batch-to-batch variations. Aptamers, an emerging class of molecules that rival antibodies have become the focus of attention over the past few decades. They are short, chemically synthesized, singlestranded oligonucleotides (DNA or RNA), possessing unique tertiary structures. Aptamers with high affinity and specificity to identify target molecules can be selected via a reliable and efficient procedure known as systematic evolution of ligands by exponential enrichment (SELEX). Collectively, aptamers are less expensive and tedious to manufacture, more stable in longterm storage and easier to synthesize, manipulate, and functionalize compared with antibodies [2]. Moreover, to ensure highly sensitive detection, noble metal nanomaterials offer an unprecedented potential in terms of signal transduction. Noble metal nanomaterials with unique physical and chemical properties (e.g., surface plasmonic effects or luminescence properties) can markedly improve the sensitivity and specificity for their applications in biosensors. With the integration of aptamers as the recognition elements, these biosensing nanoplatforms allow a promising way for cost-effective, rapid and sensitive on-site detection of microbial toxin. Some of these studies have been reviewed elsewhere, with a focus on the fabrication electrochemical aptasensors for the detection of toxins based on gold nanomaterials, or as subclasses in a more general overview of the nanomaterial-based aptasensors [3,4]. In this review, we will focus on the recent development of noble metal nanomaterial-based aptasensors for microbial toxin detection and discuss some major achievements in this field over the past few years, with the aim of improving their future performances of noble metal nanomaterial-based aptasensors in food safety examination.

\section{Noble metal nanomaterial-based aptasensors for microbial toxin detection}

Noble metal nanomaterials, such as gold nanoparticles (AuNPs), gold nanorods (AuNRs), gold nanoclusters (AuNCs), silver nanoparticles (AgNPs), silver nanoclusters (AgNCs), and bimetallic nanomaterials, offering fascinating signaling properties have been employed as sensing elements to design novel aptasensors. Other attractive characteristics, such as convenient and controllable synthesis, high biocompatibility, size-dependent optical and electronic properties, and ease of surface modification, also make them extremely suitable for developing various types of aptasensors, including colorimetric, fluorescent, surface-enhanced Raman scattering (SERS), electrochemical, and portable sensors (Table 1). In the following sections, we will introduce representative examples of each of these classes of aptasensors for microbial toxin detection.

\subsection{AuNP-based aptasensors}

\subsubsection{AuNP-based colorimetric aptasensors}

It is well known that AuNPs exhibit strong distance-dependent optical properties. Once aggregated, the color of AuNPs changes from red to blue, accompanying with a bathochromic shift of the absorption spectrum. This phenomenon is attributed to the alterations in the localized surface plasmon resonance (LSPR) absorption generated by AuNPs. As AuNPs are generally thermodynamically unstable and tend to flocculate at high ionic strength conditions, surface adsorption of single-stranded DNA (ssDNA) can protect them from salt-mediated aggregation [5]. It has become one appealing strategy extensively adopted for a label-free colorimetric aptasensing. For example, Yang and coworkers have reported a rapid and sensitive aptasensor for OTA detection (Fig. 1A) [6]. When OTA is introduced, the conformation of the OTA's aptamer switched from a random coil to a rigid G-quardruplex conformation required for target binding. AuNPs are no longer protected and aggregation occurs accompanied by a distinct color change from red to blue. This strategy has further been extended to the analysis of a variety of toxins, such as Staphylococcal enterotoxin B (SEB) [7], aflatoxin B1 (AFB1) [8], ZEN [9], and microcystin-LR (MC-LR) [10]. To achieve an effective detection, it is noteworthy that the ratio 


\begin{tabular}{|c|c|c|c|c|c|}
\hline Nanomaterials & Method & Target & Strategy & LOD & Ref. \\
\hline \multirow{20}{*}{ AuNPs } & Colorimetric & OTA & Target-induced aggregation of AuNPs & $20 \mathrm{nM}$ & [6] \\
\hline & Colorimetric & SEB & Target-induced aggregation of AuNPs & $10 \mathrm{ng} / \mathrm{mL}$ & [7] \\
\hline & Colorimetric & AFB1 & Target-induced aggregation of AuNPs & $0.025 \mathrm{ng} / \mathrm{mL}$ & [8] \\
\hline & Colorimetric & ZEN & Target-induced aggregation of AuNPs & $12.5 \mathrm{nM}$ & [9] \\
\hline & Colorimetric & MC-LR & Target-induced aggregation of AuNPs & $0.37 \mathrm{nM}$ & [10] \\
\hline & Colorimetric & MC-LR & Target-induced disassembly of AuNPs & $0.05 \mathrm{nM}$ & [51] \\
\hline & Colorimetric & OTA & Enzyme-induced aggregation of AuNPs & $5.0 \mathrm{nM}$ & [11] \\
\hline & Colorimetric & AFB1 & Catalytic DNA circuit amplification strategy & $10 \mathrm{pM}$ & [12] \\
\hline & Colorimetric & SEB & Target-regulated growth of AuNPs & $1 \mathrm{pg} / \mathrm{mL}$ & [13] \\
\hline & Colorimetric & OTA & Target-regulated growth of AuNPs & $1 \mathrm{nM}$ & [14] \\
\hline & Lateral Flow Test Strip & OTA & Competition between OTA and cDNA with aptamer & $1 \mathrm{ng} / \mathrm{mL}$ & [15] \\
\hline & Lateral Flow Test Strip & ZEN & Competition between ZEN and cDNA with aptamer & $20 \mathrm{ng} / \mathrm{mL}$ & [16] \\
\hline & Fluorescent method & OTA & FRET between FAM-aptamer and AuNP & $5 \mathrm{nM}$ & [17] \\
\hline & Electrochemical & CDTA & $\begin{array}{l}\text { Electrode was modifed AuNPs-capture DNA; aptamer-HRP was } \\
\text { used to amplify the detection signal }\end{array}$ & $1 \mathrm{nM}$ & [18] \\
\hline & Electrochemical & SEB & $\begin{array}{l}\text { Electrode was modifed AuNPs, ZrO2NPs, chitosan, and capture DNA; } \\
\mathrm{H}_{2} \mathrm{O}_{2} \text { was used as the substrate of probe DNA-HRP }\end{array}$ & $0.24 \mathrm{ng} / \mathrm{mL}$ & [19] \\
\hline & Electrochemical & FB1 & SPCE was covered by PDMS and AuNPs & $3.4 \mathrm{pg} / \mathrm{mL}$ & [22] \\
\hline & Electrochemical & PAT & Electrode was modifed with $\mathrm{ZnO}$ nanorods, AuNPs, and aptamer & $0.27 \mathrm{pg} / \mathrm{mL}$ & [23] \\
\hline & Electrochemical & AFM1 & AFM1-mediated AuNPs close proximity to SPGE & $0.9 \mathrm{ng} / \mathrm{mL}$ & [24] \\
\hline & Electrochemical & OTA & $\begin{array}{l}\text { Capture DNA, aptamer, reporter DNA-AuNP were immobilized } \\
\text { on electrode; methylene blue was used as a redox indicator }\end{array}$ & $0.75 \mathrm{pM}$ & [20] \\
\hline & Electrochemical & AFB1 & Telomerase and EXO III based signal amplification strategy & $0.6 \times 10^{-4} \mathrm{pg} / \mathrm{mL}$ & [21] \\
\hline \multirow[t]{5}{*}{ AuNRs } & LSPR-based portable method & OTA & $\begin{array}{l}\text { Aptamer-labeled AuNRs were coated on the optical fiber; aptamer-OTA } \\
\text { binding events caused the LSPR peak of AuNR red shift. }\end{array}$ & $12 \mathrm{pM}$ & [25] \\
\hline & Multicolor colorimetric method & OTA & G-quadruplex-hemin DNAzyme-mediated etching of AuNRs & $30 \mathrm{nM}$ & [26] \\
\hline & Multicolor colorimetric method & OTA & ALP-mediated metalization of AuNRs & $9 \mathrm{nM}$ & [27] \\
\hline & SERS & SEB & $\begin{array}{l}\text { Aptamer modified on the MNPs to capture SEB from the food matrix; Raman } \\
\text { reporter and aptamer immobilized AuNRs form a sandwich-like detection model }\end{array}$ & $2.2 \times 10^{-16} \mathrm{M}$ & [28] \\
\hline & Electrochemical & OTA & AuNRs modified-Au electrode; DNA tetrahedral nanostructures & $0.26 \mathrm{pg} / \mathrm{mL}$ & [29] \\
\hline AuNCs & Fluorometric & AFB1 and ZEN & FRET between dual-color AuNCs and $\mathrm{WS}_{2}$ nanosheets & $\begin{array}{l}\text { AFB1: } 0.34 \mathrm{pg} / \mathrm{mL} \text {; } \\
\text { ZEN: } 0.53 \mathrm{pg} / \mathrm{mL}\end{array}$ & [32] \\
\hline \multirow[t]{4}{*}{ AgNPs } & Fluorometric & AFB1 & FRET between fluorescent polymer dots and AgNPs & $0.3 \mathrm{pg} / \mathrm{mL}$ & [33] \\
\hline & SERS & OTA & $\begin{array}{l}\text { Target-mediated Raman reporter Cy5 labelled aptamer desorption from the } \\
\text { surface of AgNPs }\end{array}$ & $0.1 \mathrm{nM}$ & [34] \\
\hline & Electrochemical & OTA & Gold electrode covered with neutral red and aptamer-AgNPs & $0.05 \mathrm{nM}$ & [35] \\
\hline & Electrochemical & OTA & $\begin{array}{l}\text { Aptamer was immodibized on the gold electrode; AgNPs/PDANSs were used } \\
\text { as electrochemical tracer }\end{array}$ & $0.57 \mathrm{pM}$ & [36] \\
\hline \multirow[t]{4}{*}{ AgNCs } & Fluorometric & SEA & FRET between AgNCs and PPNPs & $0.3393 \mathrm{ng} / \mathrm{mL}$ & [38] \\
\hline & Fluorometric & $\mathrm{T}-2$ toxin & FRET between AgNCs and $\mathrm{MoS}_{2}$ & $0.93 \mathrm{pg} / \mathrm{mL}$ & [39] \\
\hline & Fluorometric & OTA and AFB1 & Magnetic separation and DNA-template AgNCs as the fluorophore & $\begin{array}{l}\text { OTA: } 0.2 \mathrm{pg} / \mathrm{mL} ; \\
\text { AFB1: } 0.3 \mathrm{pg} / \mathrm{mL}\end{array}$ & [41] \\
\hline & Electrochemical & OTA & $\begin{array}{l}\text { Aptamer was modified on the surface of SPGE; AgNCs metallization on } \\
\text { OTA/aptamer complex }\end{array}$ & $0.7 \mathrm{pg} / \mathrm{mL}$ & [42] \\
\hline
\end{tabular}


between aptamers and AuNPs requires careful adjustments. Typically, a least excess of free aptamers is needed to ensure an adequate protection of AuNPs against non-specific aggregation, but without jeopardizing the detection sensitivity. Recently, a sensitive colorimetric aptasensor was developed for the detection of OTA by He and coworkers based on enzyme-induced AuNP aggregation [11]. When OTA was absent, the target-binding aptamer pre-immobilized on the magnetic nanoparticles (MNPs) was hybridized to its complementary sequence possessing an enzyme at one end (cDNA-ALP). However, as the OTA was present, the duplex structure would switch to an OTA-aptamer complex together with the release of cDNA-ALP. Followed by magnetic separation to remove immobilized enzymes, the liberated enzyme (ALP) can turn over the substrate, initiating the subsequent reactions, leading to salt-mediated AuNP aggregation. The proposed method was successfully applied to determine OTA in red wine and grape juice samples, offering high sensitivity with a detection limit as low as $5.0 \mathrm{nM}$. In addition, with efficient cascade amplification strategies, enzyme-free DNA circuits could also facilitate the development of ultrasensitive toxin detection. For example, a catalytic DNA circuit was devised by Chen et al. to amplify the colorimetric signal of AuNPs for the determination of AFB1 [12]. The constructed assay is ultrasensitive, allowing the visual detection of AFB1 as low as $10 \mathrm{pM}$ without instrumentation.

Recently, a new generation of colorimetric aptasensors has been developed based on the targetregulated growth of AuNPs. According to this principle, an aptasensor was constructed by Zhou and coworkers for the detection of SEB [13]. In the absence of SEB, the guanine (G)-rich DNA oligonucleotides, which were sealed by the SEB aptamer, lost their ability to consume $\mathrm{H}_{2} \mathrm{O}_{2}$. Hence, AuNPs were synthesized in situ through the reduction of gold ions by $\mathrm{H}_{2} \mathrm{O}_{2}$, leading to a sensing solution with a red color. However, in the presence of SEB, the structure switching of the aptamer triggered the liberation of G-rich DNA oligonucleotides. The formation of G-quadruplex-based DNAzymes exhibiting a peroxidase-mimicking activity is capable of consuming $\mathrm{H}_{2} \mathrm{O}_{2}$, which in turn, producing blue-colored solutions. The linear range of this method extended from 0.1 to $500 \mathrm{ppt}$, allowing for clinical diagnosis. Similarly, another targetregulated-AuNP-growth strategy was developed by Soh et al. for OTA detection [14]. The interaction between aptamer and its target may affect the amount of aptamer adsorbed on the Au NP surface, which consequently generate AuNPs of variable 

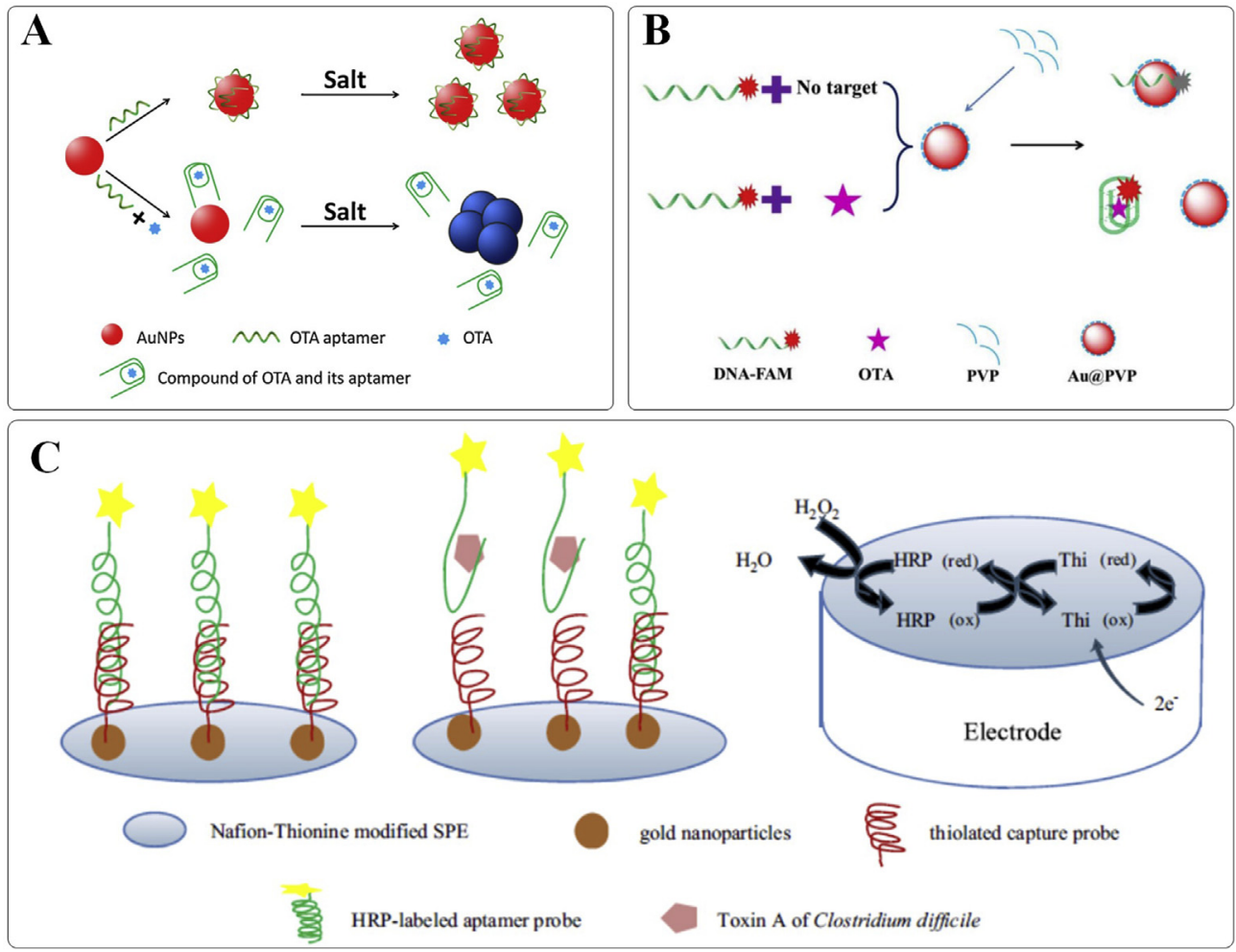

Fig. 1. (A) Schematic representation of a colorimetric aptasensor based on unmodified AuNPs for OTA detection. Adapted with permission from Ref. [6]. Copyright Elsevier, 2011. (B) Schematic representation of a fluorescent aptasensor based on AuNPs for OTA detection. Adapted with permission from Ref. [17]. Copyright Elsevier, 2018. (C) Schematic representation of an electrochemical aptasensor based on AuNPs for CDTA detection. Adapted with permission from Ref. [18]. Copyright Elsevier, 2014.

morphologies. As the OTA concentration increased, the color of the solution changed from blue to red. A visible colorimetric response was archived successfully in red wine samples for the detection of OTA at concentrations as low as $1 \mathrm{nM}$.

Due to the advantages of simplicity, portability, convenience, and low cost, AuNP-based lateral-flow test strips hold great potential for on-site foodcontamination detection. Zhou et al. developed, for the first time, an AuNP-based lateral-flow test strip for the colorimetric detection of OTA using an aptamer as the recognition element [15]. With its high sensitivity and selectivity, a visual limit of detection of $1 \mathrm{ng} \mathrm{mL}^{-1}$ of OTA was achieved with in $15 \mathrm{~min}$. Later, the designed aptamer-based strip was also successfully applied to the determination of ZEN in corn samples [16]. The above results indicate that the current technology is able to provide rapid, sensitive and reliable results for numerous food contaminants and multitudinous sample inspection.

\subsubsection{AuNP-based fluorescent aptasensors}

Due to the strong LSPR and high coefficient extinction, AuNPs can also be used as energy acceptors for constructing aptasensors based on the mechanism of Förster resonance energy transfer (FRET). For example, an aptasensor was reported by Lv et al. for OTA detection with the use of AuNPs as efficient fluorescence quenchers [17]. As shown in Fig. $1 \mathrm{~B}$, in the absence of OTA, the carboxyfluorescein (FAM)-labeled aptamer was adsorbed on the surface of the AuNPs, leading to fluorescence quenching of FAM. Upon addition of OTA, the binding event between OTA and the aptamer caused the aptamer to desorb from the surface of the AuNPs, which led to a recovery of the FAM fluorescence. This turn-on aptasensor had a detection limit of $5 \mathrm{nM}$ for OTA and was found to be to be free of interference by the sample matrix of ginger powders.

\subsubsection{AuNP-based electrochemical aptasensors}

Thanks to the high surface-to-volume ratio, high electron transfer efficiency and excellent biocompatibility, AuNPs has been extensively employed as a solid support for biomolecule immobilization to facilitate the electron transfer between the electrodes and biomolecules, giving the electrochemical devices with high sensitivity. For example, a sensitive AuNP-based electrochemical aptasensor has 
been constructed by Luo and coworkers for the detection of type-A C. difficile toxins (CDTA) (Fig. 1C) [18]. In the developed device, the electrode was modified by layer-by-layer self-assembly of Nafion-Thionine, AuNPs, capture DNA, and horseradish peroxidase (HRP)-labeled aptamer, respectively. When CDTA was present, HRPlabeled aptamer would dissociate from the electrode surface through the target binding-induced conformational change. As a result, the catalytic activity of HRP was reduced, accompanying by a decrease in the amperometric response. The detection limit was shown to be $1 \mathrm{nM}$ for CDTA with good selectivity, reproducibility, stability, and accuracy. Afterward, Deng et al. has suggested a similar strategy for the detection of SEB [19]. The electrochemical aptasensor, based on a AuNPs/ $/ \mathrm{rO}_{2} /$ chitosan-modified electrode coupled with a HPR-assisted signal amplification process, was capable of detecting SEB in a wider linear range with a much lower detection limit, providing a versatile tool in clinical diagnosis. To achieve a superior electrochemical response, an ultrasensitive electrochemical aptasensor based on the two-level cascaded signal amplification strategy was described by Yang et al. for OTA detection [20]. In the design, capture DNA, aptamers, and reporter DNA-modified AuNPs were immobilized on the electrode in a sandwiched manner. While AuNPs has been employed as the first-level signal enhancer, a great number of G-rich DNA hybridized on the AuNP surface promoted abundant methylene blue (redox indicator) anchoring, allowing a second-level signal enhancement to further amplify the initial electrochemical response. In the presence of OTA, the binding-induced conformation change enabled the release of G-rich DNA/ AuNPs from the electrode surface, thereby reducing the electrochemical response. The proposed method showed promise in the sensitive detection of OTA with a low detection limit of $0.75 \mathrm{pM}$. Another signal enhancement approach based on two-round enzymatic amplification reactions was adopted by Zheng et al. for trace detection of AFB1 [21]. The aptamer probes were immobilized on the electrode surface and hybridized with the cDNA-modified AuNPs cotethered with multiple copies of telomerase primers. Telomerase was then added to elongate the primers for abundant methylene blue anchoring, thereby enlarging the initial current signal to achieve the first round of amplification. As the target was present, the probe aptamers would undergo conformation change to release the primer-AuNP-cDNA conjugates from the sensing interface. Exonuclease III was further used to hydrolyze the resulting dsDNA probes to assist AFB1 release and recycling for the second round of amplification. Taking
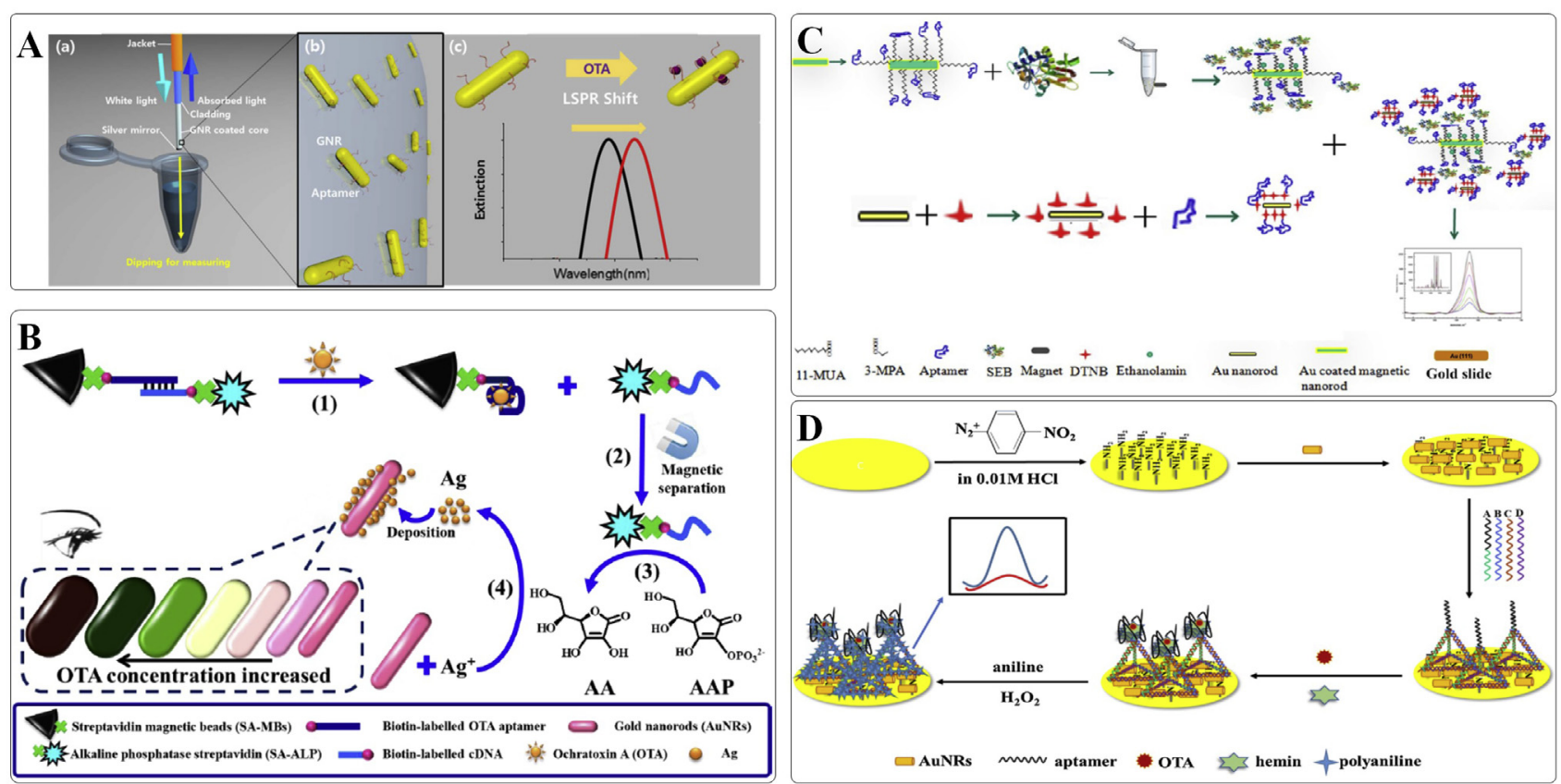

Fig. 2. (A) Schematic representation of an optical fibers based on AuNRs for OTA detection. Adapted with permission from Ref. [25]. Copyright Elsevier, 2018. (B) Schematic representation of enzyme-mediated metallization of AuNRs for multicolor colorimetric detection of OTA. Adapted with permission from Ref. [27]. Copyright Elsevier, 2020. (C) Schematic representation of a SERS aptasensor based on AuNRs for homogeneous sandwich detection of SEB. Adapted with permission from Ref. [28]. Copyright American Chemical Society, 2012. (D) Schematic of a DNA tetrahedral nanostructure-based aptasensor for OTA detection. Adapted with permission from Ref. [29]. Copyright Elsevier, 2018. 
advantage of the dual-amplification effect, this signal-off aptasensor enabled three orders of magnitude improvement in detection sensitivity, achieving an ultralow detection limit of $0.6 \times 10^{-4} \mathrm{pg} \mathrm{mL}^{-1}$ of OTA.

Moreover, a disposable aptasensing device for label-free detection of fumonisin B1 (FB1) has been reported by Ren et al. In the design, the screenprinted carbon electrode (SPCE) was equipped with a self-designed microcell. A dense decoration of AuNPs on SPCE was accomplished by an in situelectrodeposition, providing abundant binding sites for aptamer anchoring, finally enhancing the impedance signal significantly. The specific binding between FB1 and aptamer inhibited the redox probe $\left[\mathrm{Fe}(\mathrm{CN})_{6}\right]^{3-14-}$ from reaching the electrode surface. Therefore, FB1 can be easily detected by monitoring the electrochemical impedance spectroscopy of the system. The developed aptasensor presents a promising strategy to achieve facile, cost-effective, and highly sensitive detection of FB1, with a limit of detection of $3.4 \mathrm{pg} \mathrm{mL}^{-1}$ [22]. He et al. also reported an aptasensor utilizing $\mathrm{ZnO}$ nanorods for higher AuNPs and probe loading to effectively improve the electrode performance toward PAT detection [23]. Upon target binding, the formation of PAT-aptamer complex on the electrode surface would impede the electron transfer between the electrode and a redox indicator, hexacyanoferrate, causing the current to decrease. The prepared aptasensor showed satisfactory results when applied to the determination of PAT in apple juice samples. A signal-on electrochemical aptasensor was developed by Jalaliana et al. for the detection of aflatoxin M1 (AFM1) [24]. In the presence of the AFM1, the hairpin structure of the probe aptamers would undergo conformation change; thereby allowing a target-induced immobilization of AuNPs in close contact to the electrode surface. Upon the addition of redox indicators, the current signal would be increased, enabling a sensitive detection of AFM1 with a detection limit of $0.9 \mathrm{ng} \mathrm{\textrm {L } ^ { - 1 }}$. The developed aptasensor has been successfully applied to AFM1 analysis in real samples, including milk and serum samples.

\subsection{AuNR-based aptasensors}

AuNRs, a particular type of anisotropic metal NPs, exhibit two LSPR peaks, namely, a longitudinal and a transverse LSPR peak. The LSPR wavelength, especially for the longitudinal mode can be tuned over hundreds of nanometers in the visible and near-infrared regions by alterations of the aspect ratio (length/width). For this reason, AuNRs are considered as an attractive optical transducer for biosensing, in particular when a specific wavelength is desired.

\subsubsection{AuNR-based LSPR aptasensors}

Lee et al. constructed a portable LSPR aptasensor based on optical fibers for facile and rapid OTA detection [25]. In the design, the aptamer-labeled AuNRs were coated on the optical fibers and subjected to OTA analysis. As illustrated in Fig. 2A, upon target binding, the aptamer is transformed into a G-quadruplex structure, and thus, leading to a red shift of the LSPR peak. Through the sequential dipping and measurement process, OTA in grape juice can be accurately detected with a limit of detection of $12.0 \mathrm{pM}$. The developed device is simple to operate and can be applied to in-situ analysis of OTA.

\subsubsection{AuNR-based multicolor colorimetric aptasensors}

In addition to the alternation in local refractive index induced with an aptamer-target binding event, etching or metallization of AuNRs also lead to a significant modulation of the LSPR peak, turning solutions of rainbow-like colors. Based on this principle, several multicolor colorimetric methods have been developed for the visual semiquantitative determination of a variety of targets. Yu et al. established a multicolor colorimetric aptasensor for the visual detection of OTA based on the G-quadruplex-hemin DNAzyme-mediated etching of AuNRs [26]. A DNA sequence that was composed of a hemin aptamer and an OTA aptamer was designed as a recognition probe. In the absence of OTA, this ssDNA probe was cut into fragments by Exo I from the 3' end. However, in the presence of OTA, the target binding-induced structure switching can halt the Exo I-mediated digestion due to the steric hindrance. At the same time, the retained hemin aptamer can convert to its active form and exerts a peroxidase-like activity to catalyze the oxidation of $3,3^{\prime}, 5,5^{\prime}$-tetramethylbenzidine (TMB) to $\mathrm{TMB}^{2+}$. $\mathrm{TMB}^{2+}$ was capable to etch AuNR by oxidizing $\mathrm{Au}(0)$ to $\mathrm{Au}(\mathrm{I})$, causing a vivid color change for visual inspection of OTA with the detection limit of $30 \mathrm{nM}$. Tian et al. reported another multicolor colorimetric approach for OTA detection on the basis of enzyme-mediated metallization of AuNRs (Fig. 2B) [27]. With OTA binding to its aptamer, the structural switching of the aptamer enabled the release of cDNA-ALP. Following the cDNA-ALP-catalyzing reactions, the obtained products, ascorbic acids could reduce $\mathrm{Ag}^{+}$to $\mathrm{Ag}$ on the surface of AuNRs, resulting in a visible multicolor change. The assay was quite sensitive, since approximately $9 \mathrm{nM}$ OTA could be readily detected 
by the naked eye. Collectively, AuNR-based multicolor colorimetric method hold great potential for the on-site visual detection of toxins in resourcelimited regions.

\subsubsection{AuNR-based SERS aptasensors}

Plasmonic nanostructures, particularly AuNPs and AgNPs have been explored extensively as SERS substrates for decades. In general, anisotropic NPs possessing sharp tips, corners and edges give rise to intense local electromagnetic fields, providing a strong Raman enhancement for SERS. SERS with state-of-the-art performance is capable of offering extremely high sensitivity, down to the singlemolecule detection level. Temur et al. established an ultrasensitive aptasensor using AuNRs as SERS probes for SEB detection [28]. As shown in Fig. 2C, Au-coated MNPs were functionalized with the capture aptamers to separate SEB from the food matrix. Then, the aptamer-immobilized and Raman reporter-tagged AuNRs can conjunct with the captured targets via a sandwich-like manner, giving rise to a strong SERS signal. The proposed method achieved a detection limit of $2.2 \times 10^{-16} \mathrm{M}$ of SEB and is applicable to complex matrixes, such as milk, urine, and serum.

\subsubsection{AuNR-based electrochemical aptasensors}

Similar to AuNPs, AuNRs have also been widely used in electrochemical biosensors for signal amplification. An ultrasensitive aptasensor has been developed by Wei et al. for OTA detection by utilizing an AuNR-modified electrode to enhance the loading of DNA tetrahedral probe (DTP) and to promote the electron-transfer rate (Fig. 2D) [29]. DTP, as a novel type of probe, was modified with an OTA aptamer on the top vertex and three thiol groups at the other three vertices. Once assembled onto the electrode, DTP exhibited a stronger bonding with gold surfaces than the conventional ssDNA probe with just one anchor point. Moreover, the mechanical rigidity of DTP can force the aptamer to adopt an upwards orientation, thereby providing efficient molecular recognition at the sensing interface with encouraging outcomes [30]. The OTA/aptamer binding event causes a transformation of the aptamer structure into a G-quadruplex. The G-quadruplex binds to hemin to form a DNAzyme with peroxidase-mimicking activity that can catalyze the oxidation of aniline to polyaniline in the presence of $\mathrm{H}_{2} \mathrm{O}_{2}$, thereby generating an amplified current signal. The obtained aptasensor presented acceptable reproducibility and repeatability, and had high selectivity toward OTA against other mycotoxin, with the detection limit of $0.26 \mathrm{pg} \mathrm{mL}^{-1}$.

\subsection{AuNC-based aptasensors}

When the particle size of noble metals becomes comparable to the Fermi wavelength for the electron, LSPR peaks disappeared. In this ultrasmall size regime, metal nanoclusters (NCs), consisting of several to hundreds of atoms, exhibit unique molecule-like properties including discrete electronic states and size-dependent photoluminescence. So far, fluorescent metal NCs have been extensively studied for biosensing and bioimaging due to their attractive set of features, such as ultrasmall size, good biocompatibility, variable emission wavelength and excellent photostability. As an important class of noble metal NCs, AuNCs are more attractive because of their ease of synthesis and modification [31]. For example, biomoleculeprotected AuNCs with tunable fluorescent colors have been prepared based on one-pot strategy via a facile biomineralization-mimicking process [32]. After the covalent modification of AFB1 and ZEN aptamers, respectively, on the surfaces of the blue$(442 \mathrm{~nm})$ and red-emitting $(650 \mathrm{~nm})$ AuNCs, these two donor probes were attached to the $\mathrm{WS}_{2}$ nanosheet, as an efficient fluorescence quencher. Binding of the specific targets induced a concentrationdependent fluorescence recovery, allowing this dual-color turn-on nanoplatform for simultaneous detection of AFB1 and ZEN. Under optimal conditions, AFB1 and ZEN can be sensitively detected with detection limits of 0.34 and $0.53 \mathrm{pg} \mathrm{mL}^{-1}$, respectively.

The developed aptasensor presenting an accurate and reliable strategy for simultaneous recognition, holds a promising potential in multipurpose biosensing.

\subsection{AgNP-based aptasensors}

\subsubsection{AgNP-based fluorescent aptasensors}

Apart from gold, AgNPs have also attracted considerable attention for the development of biosensors. Due to their broad LSPR absorption and high extinction coefficients, particularly in the visible range, it makes them to be good candidates as efficient energy acceptors. For example, Nasirian et al. developed an aptasensor for AFB1 detection based on the FRET process between aptamerlabelled fluorescent polymer dots and AgNPs conjugated with cDNA [33]. In the absence of AFB1, DNA hybridization would bring the FRET pair in 
close proximity, witching off the fluorescence. As the aptamer bound to its target, the FRET pair was separated, resulting in recovery of fluorescence. The assay was successfully applied to the detection of AFB1 in wheat flour, with an ultralow detection limit of $0.3 \mathrm{pg} \mathrm{mL}^{-1}$.

\subsubsection{AgNP-based SERS aptasensors}

In addition, AgNPs have been widely employed as SERS substrates due to the strong electromagnetic field enhancement on and near the surface of AgNPs. Ganbold et al. developed a SERS aptasensor based on AgNPs for OTA detection [34]. The Raman reporter Cy5-labeled aptamer was adsorbed on the surface of the AgNPs, generating high SERS signals. The specific binding between the aptamer and OTA induced the departure of the SERS-tagged aptamer from the substrates, resulting in a decrease in the intensity of the SERS signals. This SERS aptasensor exhibited a low detection limit of $0.1 \mathrm{nM}$ of OTA.

\subsubsection{AgNP-based electrochemical aptasensors}

Recently, the use of AgNPs on the electrode surface led to an enhanced analytical performance due to an improvement in the regularity of the composition of the electrode surface and an enhancement of the electron transfer between the electrode and the modified biomolecules. Evtugyn et al. developed an electrochemical aptasensor based on AgNPs for OTA detection [35]. In their design, a gold electrode covered with electropolymerized neutral red and AgNPs was covalently attached with multiple copies of thiolated OTA aptamers through $\mathrm{Ag}-\mathrm{S}$ bonds. The formation of the aptamer-OTA complex resulted in switching the conformation of the aptamer, which caused an increase of the charge transfer resistance and impedimetric signal changes. The applicability of the developed aptasensor was validated in beer samples, with a detection limit of $0.05 \mathrm{nM}$ of OTA. To further improve the detection sensitivity, Zhang et al. developed an ultrasensitive electrochemical aptasensor utilizing polydopamine nanospheres (PDANSs) for signal amplification [36]. Due to the large surface area of the PDANSs, a greater amount of AgNPs was immobilized on their surface. The detection was realized by the electrochemical stripping signal of AgNPs on PDANS. After OTA binding, a target-induced combination change enabled the release of signal tags on the aptasensor surface, thereby reducing the electrochemical response signal. The proposed strategy offered a wide linearity ranges of 4 orders of magnitudes and a low detection limit of $0.57 \mathrm{pM}$ of OTA.

\subsection{AgNC-based aptasensors}

\subsubsection{AgNC-based fluorescent aptasensors}

As inspired by the nature process of biomineralization, the cytosine-rich oligonucleotide has been successfully utilized as a scaffold for the synthesis of

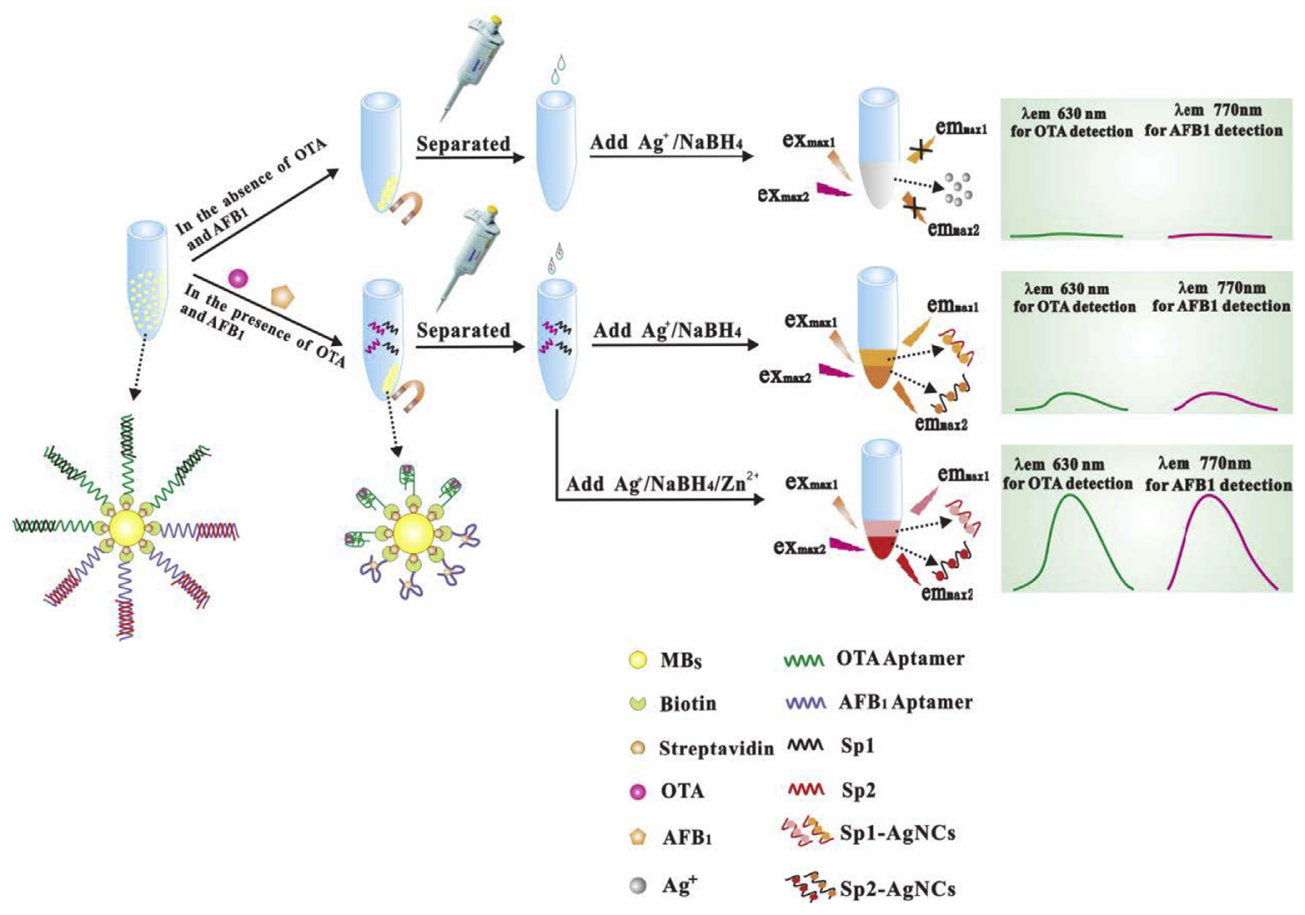

Fig. 3. Schematic representation of an aptasensor based on AgNCs for the simultaneous detection of OTA and AFB1. Adapted with permission from Ref. [41]. Copyright Elsevier, 2016. 

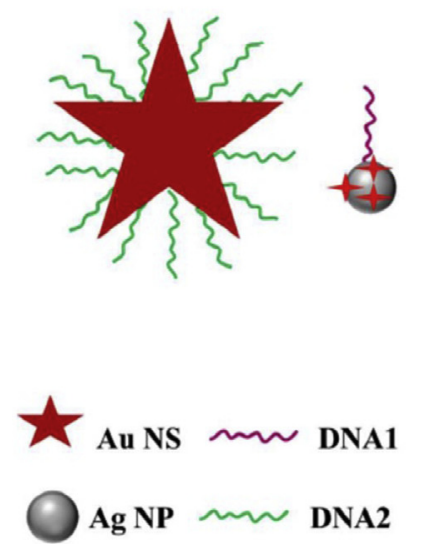
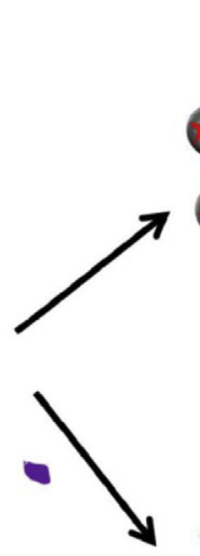

AFB1

+ Raman reporter

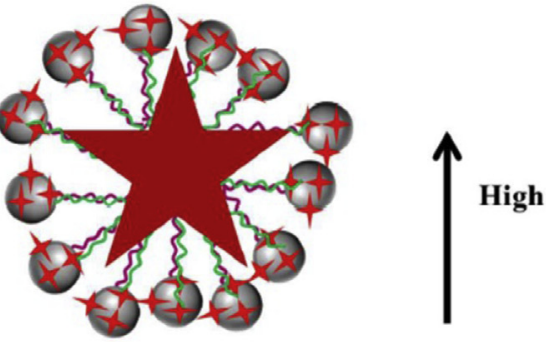

SERS

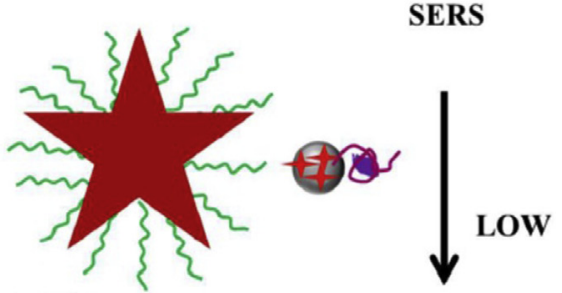

Fig. 4. Scheme of a SERS aptasensor based on AuNS core-AgNP satellites for the detection of AFB1. Adapted with permission from Ref. [44]. Copyright Royal Society of Chemistry, 2016.

fluorescent AgNCs [37]. These DNA-templated AgNCs have received great attention as novel nanotools in bioapplications, due to their strong fluorescence, excellent light stability, and good biocompatibility. It is therefore, highly promising for the construction of label-free fluorescent aptasensors based on AgNCs. By designing a chimeric oligonucleotide, which contains a domain with a 12mer cytosine for AgNC formation and a domain with an aptamer for target recognition, Zhang et al. developed a label-free aptasensor for Staphylococcal enterotoxin A (SEA) detection [38]. In their design, polypyrrole nanoparticles (PPNPs) were used as the quencher. The specific binding between SEA and the aptamer caused the aptamer-DNA-AgNCs complex to move away from the PPNPs, leading to an effective fluorescence recovery. This label-free aptasensor is very simple and cost effective, with a low detection limit of $0.3393 \mathrm{ng} \mathrm{mL}^{-1}$. A similar strategy has also been reported by Khan et al. for the detection of T-2 toxin [39]. Based on the use of AgNC and $\mathrm{MoS}_{2}$ as a FRET pair, a sensitive detection of T-2 toxin in maize and wheat samples was achieved with a low detection limit of $0.93 \mathrm{pg} \mathrm{mL}^{-1}$.

Simultaneous monitoring of various mycotoxins is essential for food safety because agricultural products and food stuff are often contaminated with multiple mycotoxins. Delightfully, the fluorescent emission of AgNCs are finely tunable by adjusting the sequence and length of the DNA template [40]. Thereofore, AgNCs hold great potential for the simultaneous detection of different mycotoxins.
Based on this principle, Zhang et al. developed a fluorescent aptasensor for the simultaneous detection of OTA and AFB1 [41]. As shown in Fig. 3, an OTA aptamer and an AFB1 aptamer were immobilized on the surface of MNPs. Signal probes 1 and 2 were designed not only to be complementary to the OTA and AFB1 aptamers, respectively, but also to be used as templates to form AgNCs with different maximum emission wavelengths. Moreover, the addition of $\mathrm{Zn}^{2+}$ was found to increase the fluorescence intensity of the DNA-scaffolded AgNCs, and thereby improving the detection sensitivity. The detection limits of OTA and AFB1 was approximately 0.2 and $0.3 \mathrm{pg} \mathrm{mL}^{-1}$, respectively.

\subsubsection{AgNC-based electrochemical aptasensors}

Based on the fact that catalytic silver deposition (termed as the silver enhancement) can provide further signal enhance, silver-metallized aptamers have also been devoted to the design of electrochemical aptasensor for the ultrasensitive detection of OTA [42]. As a result of target binding, aptamers on the electrode surface would self-assembled into a G-quadruplex structures. After the introduction of Exo I to digest the randomly coiled unbound aptamers, chemical and electrochemical reduction processes were initiated to generate silver metallized aptamers for voltammetric detection. The combination of Exo-I-mediated background-signal suppression and AgNC-mediated signal amplification leads to a label-free, sensitive, specific, and "turn-on" electrochemical aptasensor for the precise determination of OTA at trace levels. 


\subsection{Bimetallic nanomaterial-based aptasensors}

\subsubsection{Bimetallic nanomaterial-based SERS aptasensors}

As an ultrasensitive analytical technique, SERS has been extensively applied for trace microbial toxin detection to ensure food safety. In general, an average enhancement factor ranges between $10^{4}$ and $10^{8}$ was reported for metallic NPs made of gold, silver, and copper [43]. More recently, it has been found that gold-silver bimetallic nanomaterials have the ability to further improve the SERS signals compared to their mono-metallic gold or silver counterparts. This phenomenon is attributed to the tunability of the SPR band and the synergistic effect of these metals. For instance, $\mathrm{Li}$ et al. reported the use of heterogeneous gold nanostar (AuNS) core-AgNP satellite assemblies as SERS-active sensors for ultrasensitive detection of AFB1 [44]. The Raman reporter was modified on the surface of AgNP satellites. As shown in Fig. 4, in the absence of AFB1, hybridization occurred between the aptamermodified AgNP satellites and cDNA-modified AuNS. Formation of the core-satellite assemblies give rise to an enhanced SERS signal. However, the specific binding of AFB1 to the aptamer caused the release of $\mathrm{Ag}$ satellites, resulting in a decreased SERS signal. The developed SERS aptasensor showed a high sensitivity for AFB1 with a detection limit of $0.48 \mathrm{pg} \mathrm{mL}^{-1}$. A variety of core-satellite assemblies have also been fabricated for ultrasensitive detection; for example, e.g., an aptasensor based on Au nanoflower core-AgNP satellites was developed for the detection of MC-LR [45]. Similarly, the SERS-active assemblies exhibited amplified SERS signals, enabling a sensitive detection of trace level of MC-LR based on the target-induced disassembly process.

Not only the heterogeneous core-satellite assemblies can produce sensitive SERS signals, gold-silver core-shell structures could also serve as excellent SERS substrates for target detection with high sensitivity. Based on this principle, various aptasensors have been developed. Yang et al. designed gold nanotriangle (AuNT)-silver core-shell nanomaterials (AuNT@Ag) for the sensitive and selective SERS detection of AFB1 [46]. To achieve AFB1 detection, aptamer-MNP and aptamer-AuNT@Ag were used as capture probe and reporter probe, respectively. Under optimized conditions, a detection limit of $0.54 \mathrm{pg} \mathrm{mL}^{-1}$ was obtained. Another aptasensor based on core-shell nanomaterials composed of $\mathrm{Au}_{(\text {core) }} @ \mathrm{Au}-\mathrm{Ag}_{\text {(shell) }}$ was also constructed by Shao et al. for the detection of OTA [47]. In this strategy, cDNA-MNP and aptamer- $\mathrm{Au}_{\text {(core) }} @ \mathrm{Au}-\mathrm{Ag}_{\text {(shell) }}$ were prepared. Due to the high binding affinity between OTA and its aptamer, the presence of OTA induced cDNA-MNP to break away from aptamer- $\mathrm{Au}_{\text {(core) }} @ \mathrm{Au}-\mathrm{Ag}_{\text {(shell) }}$. And a significant change in Raman intensity was observed. An ultralow detection limit of $0.004 \mathrm{ng} \mathrm{mL}^{-1}$ was achieved successfully in red wine samples. Collectively, in comparison to the monometallic counterparts, these heterogeneous core-shell nanostructures possessing built-in nanogap hotspots have shown superior SERS signal amplification, holding great potential for ultrasensitive detection.

\subsubsection{Bimetallic nanomaterial-based electrochemical aptasensors}

In addition to the LSPR, other physicochemical characteristics such as catalytic and electrocatalytic properties of bimetallic nanomaterials may also be tremendously different from those of the parent metals. Alloyed structures, in particular, often result in a tunable electrochemical activity, attributing to the synergy effect of different electronic structure, achieved by gradually varying the composition [48]. Ji et al. developed a palladium-platinum nanoparticle (Pd-PtNP)based aptasensor for ZEN detection [49]. In their design, a glassy carbon electrode (GCE) was modified with spherical $\mathrm{Au}-\mathrm{PANI}-\mathrm{Au}$ (PANI: polyaniline) nanocomposites to increase the conductivity of the electrode and to provide more aptamer-binding sites. The cDNA-modified sakura-shaped $\mathrm{Cu} @ \mathrm{~L}-\mathrm{Glu} / \mathrm{Pd}-\mathrm{PtNPs}$ was employed as a signal tag. The hybridization reaction between the aptamer and cDNA brought the $\mathrm{Cu} @ \mathrm{~L}-\mathrm{Glu} / \mathrm{Pd}-\mathrm{PtNPs}$ close to the GCE surface, which produced obvious electrochemical signals. After the specific ZEN-aptamer binding, cDNA-Cu@L-Glu/Pd-PtNPs was released from the GCE, resulting in a significant signal decrease. Through the effective signal amplification mediated by $\mathrm{Cu} @ \mathrm{~L}-\mathrm{Glu} / \mathrm{Pd}-\mathrm{PtNPs}$, an ultralow detection limit of $0.45 \mathrm{fg} \mathrm{mL}^{-1}$ was achieved. By utilizing rGO-TEPA-Au@PtNRs as the signal tag, Zhong et al. developed another novel aptasensor for T-2 toxin detection [50]. Similarly, the high catalytic ability of Au@PtNRs enabled an efficient signal amplification, leading to a sensitive detection of T-2 toxin as low as $1.79 \mathrm{fg} \mathrm{mL}^{-1}$. Collectively, bimetallic nanomaterials which demonstrated superior catalytic and electrocatalytic activities are promising signal enhancers in electrochemical aptasensors. Such devices provide high sensitivity, representing a promising strategy for the detection of small trace toxins in food samples. 


\section{Conclusion and outlook}

Aptamers, also known as chemical antibodies, play an increasingly important role in the molecular recognition of microbial toxins because of their multiple advantages including high binding affinity, high specificity, high stability, repeatable synthesis and modification, predictable structure, and flexible amplification strategies that are easy to develop. At the same time, the outstanding physical and chemical properties of metallic nanomaterials make them excellent candidates for signal transduction. Therefore, the combination of aptamers and nanomaterials holds great potential for microbial toxin detection.

Despite a numerous aptasensors have been developed by incorporating noble metal nanomaterials for selective and sensitive detection of food toxin, most of the noble metal nanomaterial-based aptasensors developed up to date are still at the experimental stage. There are abundant challenges that remain to be overcome before it can be employed for practical detection applications. For instance, due to the complex food matrices, appropriate attention needs to be paid to the non-specific adsorption of non-target molecules on nanomaterials, which might lead to false positive signals. Moreover, some noble metal nanomaterial-based aptasensors still rely on advanced precision instruments and professional operators, which limit their widespread use in resource-limited settings.

Nevertheless, noble metal nanomaterial-based aptasensors are a promising tool for microbial toxin monitoring. As further research progresses, we will witness a thriving and prosperous development of noble metal nanomaterial-based aptasensors for portable and on-site detection of food microbial toxin in the future.

\section{Declaration of competing interest}

None.

\section{Acknowledgements}

We appreciate financial support from the National Citrus Engineering Research Center (NCERC2019007) and the National Key Research and Development Program of China (No. 2019YFC1605604). We also appreciate financial support from the Ministry of Science and Technology (108-2811-M-007-557, 108-2638-M-002 -001 -MY2) of Taiwan, ROC.

\section{References}

[1] Somerville HJ, MICROBIAL TOXINS. Ann N Y Acad Sci 1973;217:93-108.
[2] Song SP, Wang LH, Li J, Zhao JL, Fan CH. Aptamer-based biosensors. Trac Trends Anal Chem 2008;27:108-17.

[3] Rhouati A, Bulbul G, Latif U, Hayat A, Li ZH, Marty JL. Nano-aptasensing in mycotoxin analysis: recent updates and progress. Toxins 2017;9:23.

[4] Negahdary M. Electrochemical aptasensors based on the gold nanostructures. Talanta 2020;216:23.

[5] Li HX, Rothberg L. Colorimetric detection of DNA sequences based on electrostatic interactions with unmodified gold nanoparticles. PNAS USA 2004;101:14036-9.

[6] Yang C, Wang Y, Marty JL, Yang XR. Aptamer-based colorimetric biosensing of Ochratoxin A using unmodified gold nanoparticles indicator. Biosens Bioelectron 2011;26: 2724-7.

[7] Liu A, Zhang Y, Chen W, Wang X, Chen F. Gold nanoparticle-based colorimetric detection of staphylococcal enterotoxin B using ssDNA aptamers. Eur Food Res Tech 2013;237:323-9.

[8] Luan YX, Chen ZB, Xie G, Chen JY, Lu AX, Li C, et al. Rapid visual detection of aflatoxin $\mathrm{B} 1$ by label-free aptasensor using unmodified gold nanoparticles. J Nanosci Nanotechnol 2015; 15:1357-61.

[9] Zhang Y, Lu T, Wang Y, Diao C, Zhou Y, Zhao L, et al. Selection of a DNA aptamer against zearalenone and docking analysis for highly sensitive rapid visual detection with labelfree aptasensor. J Agric Food Chem 2018;66:12102-10.

[10] Li X, Cheng R, Shi H, Tang B, Xiao H, Zhao G. A simple highly sensitive and selective aptamer-based colorimetric sensor for environmental toxins microcystin-LR in water samples. J Hazard Mater 2016;304:474-80.

[11] He Y, Tian F, Zhou J, Zhao Q, Fu R, Jiao B. Colorimetric aptasensor for ochratoxin A detection based on enzymeinduced gold nanoparticle aggregation. J Hazard Mater 2019: 121758.

[12] Chen J, Wen J, Zhuang L, Zhou S. An enzyme-free catalytic DNA circuit for amplified detection of aflatoxin B1 using gold nanoparticles as colorimetric indicators. Nanoscale 2016;8:9791-7.

[13] Zhou D, Xie G, Cao X, Chen X, Zhang X, Chen H. Colorimetric determination of staphylococcal enterotoxin $B$ via DNAzyme-guided growth of gold nanoparticles. Microchim Acta 2016;183:2753-60.

[14] Soh JH, Lin Y, Rana S, Ying JY, Stevens MM. Colorimetric detection of small molecules in complex matrixes via targetmediated growth of aptamer-functionalized gold nanoparticles. Anal Chem 2015;87:7644-52.

[15] Zhou WL, Kong WJ, Dou XW, Zhao M, Ouyang Z, Yang MH. An aptamer based lateral flow strip for on-site rapid detection of ochratoxin $\mathrm{A}$ in Astragalus membranaceus. J Chromatogr B 2016;1022:102-8.

[16] Wu S, Liu L, Duan N, Li Q, Zhou Y, Wang Z. Aptamer-based lateral flow test strip for rapid detection of zearalenone in corn samples. J Agric Food Chem 2018;66:1949-54.

[17] Lv L, Jin Y, Kang X, Zhao Y, Cui C, Guo Z. PVP-coated gold nanoparticles for the selective determination of ochratoxin $A$ via quenching fluorescence of the free aptamer. Food Chem 2018;249:45-50.

[18] Luo P, Liu Y, Xia Y, Xu HJ, Xie GM. Aptamer biosensor for sensitive detection of toxin A of Clostridium difficile using gold nanoparticles synthesized by Bacillus stearothermophilus. Biosens Bioelectron 2014;54:217-21.

[19] Deng RN, Wang L, Yi GZ, Hua EH, Xie GM. Target-induced aptamer release strategy based on electrochemical detection of staphylococcal enterotoxin B using GNPs-ZrO2-Chits film. Colloids Surf, B 2014;120:1-7.

[20] Yang XW, Qian J, Jiang L, Yan YT, Wang K, Liu Q, et al. Ultrasensitive electrochemical aptasensor for ochratoxin A based on two-level cascaded signal amplification strategy. Bioelectrochemistry 2014;96:7-13.

[21] Zheng WL, Teng J, Cheng L, Ye YW, Pan DD, Wu JJ, et al. Hetero-enzyme-based two-round signal amplification strategy for trace detection of aflatoxin B1 using an electrochemical aptasensor. Biosens Bioelectron 2016;80:574-81. 
[22] Ren C, Li H, Lu X, Qian J, Zhu M, Chen W, et al. A disposable aptasensing device for label-free detection of fumonisin B1 by integrating PDMS film-based micro-cell and screen-printed carbon electrode. Sensor Actuator B Chem 2017;251:192-9.

[23] He B, Dong X. Aptamer based voltammetric patulin assay based on the use of $\mathrm{ZnO}$ nanorods. Microchim Acta 2018;185: 462.

[24] Jalalian SH, Ramezani M, Danesh NM, Alibolandi M, Abnous K, Taghdisi SM. A novel electrochemical aptasensor for detection of aflatoxin M-1 based on target-induced immobilization of gold nanoparticles on the surface of electrode. Biosens Bioelectron 2018;117:487-92.

[25] Lee B, Park JH, Byun JY, Kim JH, Kim MG. An optical fiberbased LSPR aptasensor for simple and rapid in-situ detection of ochratoxin A. Biosens Bioelectron 2018;102:504-9.

[26] Yu X, Lin Y, Wang X, Xu L, Wang Z, Fu F. Exonucleaseassisted multicolor aptasensor for visual detection of ochratoxin A based on G-quadruplex-hemin DNAzymemediated etching of gold nanorod. Microchim Acta 2018; 185:259.

[27] Tian F, Zhou J, Fu R, Cui Y, Zhao Q, Jiao B, et al. Multicolor colorimetric detection of ochratoxin A via structure-switching aptamer and enzyme-induced metallization of gold nanorods. Food Chem 2020:126607.

[28] Temur E, Zengin A, Boyaci IH, Dudak FC, Torul H, Tamer U. Attomole sensitivity of staphylococcal enterotoxin B detection using an aptamer-modified surface-enhanced Raman scattering probe. Anal Chem 2012;84:10600-6.

[29] Wei M, Zhang W. Ultrasensitive aptasensor with DNA tetrahedral nanostructure for Ochratoxin A detection based on hemin/G-quadruplex catalyzed polyaniline deposition. Sensor Actuator B Chem 2018;276:1-7.

[30] Dong S, Zhao R, Zhu J, Lu X, Li Y, Qiu S, et al. Electrochemical DNA biosensor based on a tetrahedral nanostructure probe for the detection of avian influenza A (H7N9) virus. ACS Appl Mater Interfaces 2015;7:8834-42.

[31] Sun J, Jin Y. Fluorescent Au nanoclusters: recent progress and sensing applications. J Mater Chem C 2014;2:8000-11.

[32] Khan IM, Niazi S, Yu Y, Mohsin A, Mushtaq BS, Iqbal MW, et al. Aptamer induced multicolored AuNCs-WS2 "turn on" FRET nano platform for dual-color simultaneous detection of AflatoxinB(1) and zearalenone. Anal Chem 2019;91:14085-92.

[33] Nasirian V, Chabok A, Barati A, Rafienia M, Arabi MS, Shamsipur M. Ultrasensitive aflatoxin B1 assay based on FRET from aptamer labelled fluorescent polymer dots to silver nanoparticles labeled with complementary DNA. Microchim Acta 2017;184:4655-62.

[34] Ganbold E-O, Lee CM, Cho E-M, Son SJ, Kim S, Joo S-W, et al. Subnanomolar detection of ochratoxin A using aptamer-attached silver nanoparticles and surface-enhanced Raman scattering. Anal Methods 2014;6:3573-7.

[35] Evtugyn G, Porfireva A, Sitdikov R, Evtugyn V, Stoikov I, Antipin I, et al. Electrochemical aptasensor for the determination of ochratoxin $\mathrm{A}$ at the $\mathrm{Au}$ electrode modified with $\mathrm{Ag}$ nanoparticles decorated with macrocyclic ligand. Electroanalysis 2013;25:1847-54.

[36] Zhang J, Yang K, Chen L. In situ deposition of silver nanoparticles on polydopamine nanospheres for an ultrasensitive electrochemical aptasensor of ochratoxin A. J Electrochem Soc 2019;166:H182-6.
[37] Ritchie CM, Johnsen KR, Kiser JR, Antoku Y, Dickson RM, Petty JT. Ag nanocluster formation using a cytosine oligonucleotide template. J Phys Chem C 2007;111:175-81.

[38] Zhang X, Khan IM, Ji H, Wang Z, Tian H, Cao W, et al. A label-free fluorescent aptasensor for detection of staphylococcal enterotoxin A based on aptamer-functionalized silver nanoclusters. Polymers 2020;12.

[39] Khan IM, Zhao S, Niazi S, Mohsin A, Shoaib M, Duan N, et al. Silver nanoclusters based FRET aptasensor for sensitive and selective fluorescent detection of T-2 toxin. Sensor Actuator B Chem 2018;277:328-35.

[40] Richards CI, Choi S, Hsiang J-C, Antoku Y, Vosch T, Bongiorno $\mathrm{A}$, et al. Oligonucleotide-stabilized $\mathrm{Ag}$ nanocluster fluorophores. J Am Chem Soc 2008;130:5038-9.

[41] Zhang J, Xia Y-K, Chen M, Wu D-Z, Cai S-X, Liu M-M, et al. A fluorescent aptasensor based on DNA-scaffolded silver nanoclusters coupling with Zn(II)-ion signal-enhancement for simultaneous detection of OTA and AFB1. Sensor Actuator B Chem 2016;235:79-85.

[42] Suea-Ngam A, Howes PD, Stanley CE, deMello AJ. An exonuclease I-assisted silver-metallized electrochemical aptasensor for ochratoxin A detection. ACS Sens 2019;4:1560-8.

[43] Tian ZQ, Ren B, Wu DY. Surface-enhanced Raman scattering: from noble to transition metals and from rough surfaces to ordered nanostructures. J Phys Chem B 2002;106: 9463-83.

[44] Li AK, Tang LJ, Song D, Song SS, Ma W, Xu LG, et al. A SERS-active sensor based on heterogeneous gold nanostar core-silver nanoparticle satellite assemblies for ultrasensitive detection of aflatoxinB1. Nanoscale 2016;8:1873-8.

[45] Zhao Y, Yang X, Li H, Luo Y, Yu R, Zhang L, et al. Au nanoflower-Ag nanoparticle assembled SERS-active substrates for sensitive MC-LR detection. Chem Commun 2015, 51:16908-11.

[46] Yang MX, Liu GK, Mehedi HM, Ouyang Q, Chen QS. A universal SERS aptasensor based on DTNB labeled GNTs/ $\mathrm{Ag}$ core-shell nanotriangle and $\mathrm{CS}-\mathrm{Fe} 3 \mathrm{O} 4$ magnetic-bead trace detection of Aflatoxin B1. Anal Chim Acta 2017;986: 122-30.

[47] Shao B, Ma X, Zhao S, Lv Y, Hun X, Wang H, et al. Nanogapped Au-(core) @ Au-Ag-(shell) structures coupled with $\mathrm{Fe} 3 \mathrm{O} 4$ magnetic nanoparticles for the detection of Ochratoxin A. Anal Chim Acta 2018;1033:165-72.

[48] Deng Y-J, Tian N, Zhou Z-Y, Huang R, Liu Z-L, Xiao J, et al. Alloy tetrahexahedral $\mathrm{Pd}-\mathrm{Pt}$ catalysts: enhancing significantly the catalytic activity by synergy effect of high-index facets and electronic structure. Chem Sci 2012;3:1157-61.

[49] Ji X, Yu C, Wen Y, Chen J, Yu Y, Zhang C, et al. Fabrication of pioneering 3D sakura-shaped metal-organic coordination polymers Cu@L-Glu phenomenal for signal amplification in highly sensitive detection of zearalenone. Biosens Bioelectron 2019;129:139-46.

[50] Zhong H, Yu C, Gao R, Chen J, Yu Y, Geng Y, et al. A novel sandwich aptasensor for detecting T-2 toxin based on rGOTEPA-Au@Pt nanorods with a dual signal amplification strategy. Biosens Bioelectron 2019;144:111635.

[51] Wang F, Liu S, Lin M, Chen X, Lin S, Du X, et al. Colorimetric detection of microcystin-LR based on disassembly of orient-aggregated gold nanoparticle dimers. Biosens Bioelectron 2015;68:475-80. 